\title{
The Legal System of Electronic Check in Jordanian Law
}

\author{
Researcher \\ Dr. Bassam Mostafa Tubaishat \\ Associate Professor / Jordan / Irbid National University, Faculty of Law
}

\begin{abstract}
The electronic check is one of the electronic payment methods at present, where the electronic check is equivalent to the normal check in terms of the mandatory data included in the traditional check, but the method of the electronic check is different, depending on the electronic method in which it has acquired different privacy. It is an electronic data message signed with a special signature that is the electronic signature, so the Jordanian legislator must provide explicit and clear texts regarding the electronic check to enhance confidence in dealing therewith.
\end{abstract}

Keywords: electronic money, electronic check, electronic payment methods, electronic commerce, electronic signature.

DOI: $10.7176 / J L P G / 85-16$

Publication date:May $31^{\text {st }} 2019$

\section{Introduction}

With the continuous evolution of banking techniques, the world has witnessed the use of electronic methods of performance in settling the financial transactions among parties and their use in electronic shopping on the Internet. Since, these methods have the advantage of low cost compared to the cost of traditional checks on the one hand, and the opportunities that they make available to reach wider markets, on the other.

One of the most prominent methods of e-payment is what is known as electronic checks, which is a new tool for e-payment, and an outcome of the technological revolution as a method of settling financial transactions and concluding transactions through the Internet. This method is an effective solution to many of the disputes and risks surrounding the handwritten check that is a payment method facing many problems due to the possibility of a lack of the value balance or does not meet a mandatory element that must be included in the check as provided by the Commercial Paper Regulations.

\section{Objectives of the Study}

The study aims to identify the electronic check as a new concept to remove the surrounding ambiguity because it is performed by electronic method to form a clear image of the manner to create the check and its trading mechanism. In addition, to know whether this check is subject to the same provisions that apply to the traditional check or is subject to particular legal regulations. The novelty of the electronic check and its entry into the business as a new concept has raised some confusion and ambiguity about the extent to which this check can be accepted in the scope of dealing either in terms of endorsement or in terms of its ability to discharge from obligations.

\section{Problem of the Study}

The problem of the study is centered on the creation of the electronic check, its mechanism of trading, how it is created, how it is confirmed, and whether it has a legal argument in paying the obligations. All these questions and others will be discussed.

\section{Methodology of the study}

The analytical method will be adopted by providing the information in the field of research and commenting on it if there is a need for comment in the light of the provisions of the Jordanian Electronic Transactions Law No. 15 of 2015 and the Jordanian Commercial Code No. 12 of 1966 and its amendments.

\section{Study Plan}

This study will be divided into three sections:

1 - The preliminary topic: creation of electronic check.

2 - The first topic: trading of the electronic check.

3 - The second topic: the legal force of the electronic check.

\section{The preliminary topic: creation of electronic check}

Most of the jurisprudence tend to consider the electronic check equivalent to the traditional check, where the fundamental difference between them is the electronic method used to edit the electronic check, and accordingly this topic will be divided into two requirements as follows: 
The first requirement: the parties to the electronic check and its mandatory data.

The second requirement: the procedures for creating the electronic check

The first requirement: the parties to the electronic check and its mandatory data.

First: the parties to the electronic check:

Customer: is the drawer who has an account at the paying bank.

Beneficiary: is the seller who wants to obtain the check value.

The bank: is the drawee on which the client is creating the check, and the beneficiary may have an account therein, i.e. the same bank is the drawee or may be a different bank. (Nabil Al-Arabi, P 67).

Second: Mandatory data in the electronic check:

The electronic check includes the same mandatory data as the traditional check and the electronic method is only the tool used to create the data. These data are:

1. The word "check" on the bond: the word "check" must be mentioned in the check and written in the same language as the check, under penalty of invalidity of the instrument as a check and its transformation into a normal bond. (Elias Haddad, 2009, P 427).

The wisdom of mentioning the word check in this bond is to distinguish it from other commercial bonds, especially the electronic bill of exchange payable on sight.

2. Not pending order on the condition to pay a certain amount of money: The electronic check must include a pending order on the condition of the drawer to the drawee to pay a certain amount of money to a third person. This order must be free from any condition of any kind whatsoever as this is contrary with the check function as a payment tool replacing money.

3. The drawee is the person assigned to pay the value of the check and in respect of the Jordanian legislation, the drawee is always a bank, and since the check is payable on sight, there is no need to mention the date of maturity contrary to the bill of exchange and the promissory note. (Nadia Fadil, 2006, P 113).

4. Date of the check creation and its place of creation: The date of the check creation is mandatory. The drawer may put an incorrect date in order to take advantage of the specified period of payment, but there may be a question about whether the date of the electronic check can be forged?

In fact, there is a difficulty in this. The reason is that when the check is signed with the private key, the content of the check is encrypted so that it cannot be changed or modified by anyone except by the person who signed it. Any signatory on the check, whether the drawer or the endorser, can authenticate the date of issuance, by sending it to the authentication authority that registers the date and signs it, then returns it to its sender.

It should be noted that the check free from the date of creation turns into a simple bond (Samer Sulaiman, 2013, P 111). In addition, the place of the check creation is a mandatory data in the check. If it is not mentioned, the check is created in the place next to the drawer.

Signature of the drawer: through the signature of the drawer, it is possible to identify the issuer of the check. The traditional check signature is marked by the drawer handwritten signature, but if the drawer is illiterate he is allowed to sign with his stamp or fingerprint (Jacques Hakim, 1996, p. 247). While, the electronic check is signed electronically and often using the technology of private and public keys. The beneficiary receives the private key after receiving the check and sends it to his financial institution, which has a copy of the public key so that it can understand the message (electronic check) and exchange it with the withdrawn financial institution (William $\mathrm{s}$ Davis and john benamati, 2003, p 285).

From the above, we find that the electronic check is similar to the traditional counterpart in terms of mandatory data, but it is characterized by the fact that the name of the beneficiary is mandatory as well as the difference in terms of signature and the lack of material presence of the place of payment.

The second requirement: the procedures for creating the electronic check

The online business assumes that both the trader and the customer have an account with a financial institution that is often an electronic bank. This institution should also include a database that contains the digital signatures and data of its customers so that it can verify the identity of the signatory. If the transaction is concluded and the customer is asked to pay the price, the check will be issued according to the following steps:

1. The customer shall fill out the check electronically, including all the required data in the check, and sign it with his own key, so that the edited data will be encrypted and then attach his general key certificate thereto.

2. The electronic check shall be traded from the drawer to the beneficiary where the drawer signs the check electronically after being created, then sends it electronically to the beneficiary who in turn receives the check, signs it electronically as the beneficiary and sends it to his financial institution.

3. The electronic check shall be exchanged between the financial institution of the customer and the financial institution of the beneficiary.

4. The financial institution of both the customer and the beneficiary shall review the check and verify the authenticity of the signatures and their validity, in addition to checking the availability of the balances 
and set off between them.

5. The amount shall be credited to the account of the seller (beneficiary) at the consumer's account (the customer) and each of them shall be notified of the transaction (Essam Matar, 2009, p. 73).

6. The beneficiary may ensure that the check value is transferred by monitoring the movement of his account directly on line (Dave chaffy, 2006, p39).

The first topic: trading of the electronic check.

Due to the lack of special provisions for the electronic check, how to endorse it and since the electronic check, is equivalent to the traditional check, we will apply the provisions of the traditional check to find out the suitability thereto or not.

Accordingly, we will divide this topic into two requirements:

The first requirement: provisions of traditional check endorsement

The second requirement: the privacy of the electronic check endorsement

The first requirement: provisions of traditional check endorsement

The traditional check varies depending on how it is issued

1. Nominal check: if the check is issued in the name of a specific person, it accepts the endorsement whether explicitly stating the condition to the order of or not provided therefor. In both cases, the trading is performed by endorsement. The check holder can only sign the check to transfer the fixed right to him (Aziz al-Ukaili, 2010, pp. 247-251).

2. Nominal check with the condition not to the order of: if the check is issued in the name of a specific person and expressly included the condition not to the order of or a phrase negating a condition for an order, in this case the check is not transferred by endorsement, but by following the procedure of the right transfer according to the civil law and the consequent effects(Aziz al-Ukaili, p. 250).

3. Check for the holder: This is the check that is released to pay to the holder or to a certain person with the mention of a phrase or to the holder, and is traded in this case by handling (delivery). It is considered as a movable and the possession rule shall be applied to the movable holder's bond (Elias Haddad, p. 217.) The second requirement: the privacy of the electronic check endorsement

After we have shown how to endorse the traditional check and if we want to apply this to the electronic check, we find that there is no possibility for endorsement of the electronic check except in the case of the nominal check. Since the name of the beneficiary and its signature are necessary in electronic checks, therefore there is no check for the holder.

The question that comes to mind is whether the electronic check has a back to write thereon the wording of the endorsement, Article (143) of the Jordanian Commercial Law No. (12) of 1966 and its amendments did not require any endorsement on the back of the check except for the endorsement in blank, i.e. the endorsement in which the name of the endorsee is not written. In this case, the endorsement must be on the back of the check because the presence of the signature only on the recto of the check may be used as a reference. Therefore, there is no endorsement of the holder or blank in the electronic check because the name of the beneficiary must be mentioned and signed as one of the necessary data. So the electronic check does not need to have a back (Samer Sulaiman, p. 131).

The electronic check is an encrypted data message sent by the drawer to the beneficiary who can open it using the public key without modification. Here we confront a problem that is, can the beneficiary write the endorsement formulation on the electronic check, while retaining the data and the signature placed by the drawer?( Samer Sulaiman, p. 134).

Here we suggest some solutions, including:

1. The banks should put in place new electronic forms allowing addition of electronic checks and signatures, while preserving the basic data and the drawer signature, thus becoming more like a paper check and thus the beneficiary can endorse it.

2. The Jordanian legislator did not require writing the endorsement form on the check, which can be written on another paper attached thereto. Article (143) of the Jordanian Commercial Code stipulates that when the beneficiary receives a data message (electronic check), he can open a new data message in which the endorsement form will be written and the check will be added thereto. He signs this letter with his own signature, encode it and send it to the endorsee and in this case the new data message is considered as a paper attached to the check.

Can an electronic check be created for its holder?

Article (228) of the Jordanian Commercial Code does not require mentioning the name of the beneficiary in the traditional check. Therefore, a check can be created for its holder. However, it is different in the electronic check so that the name and signature of the beneficiary must be mentioned. The beneficiary when receiving the check and wanted to cash it at the drawee bank must sign it with his electronic signature as the beneficiary intended by the drawer and then send the check to the bank that has a registration of the electronic signature of the beneficiary within its database. Therefore, it is confirmed that he is the owner of the right. Because of this 
mechanism, the drawer or endorser is prevented to create a check for the holder.

Accordingly, when there are mechanisms in the establishment of the check does not require mentioning the name of the beneficiary with the availability of a mechanism protecting the electronic check of fraud or copying, there is nothing that prevents the release for the holder as the traditional check.

The second topic: the legal force of the electronic check

The emergence of the electronic check has raised some mistrust of this new type of payment. Perhaps the main reason for the mistrust is the ignorance of some people about the extent to which this check has legal authority to meet the obligations and how to prove that payment, especially in the absence of physical presence of documents. This section will be divided into two requirements as follows:

The first requirement: authentic electronic check

The second requirement: proving the payment of the electronic check

The first requirement: authentic electronic check

The traditional check has a discharging force, i.e. it has a complete argument to pay between its parties on the contrary to money that has legal discharging force towards all (Musa Al Ameri, 2003, p. 87). But for the electronic check, Article 6 of the Model Law on Electronic Commerce issued by the United Nations Commission on the International Commercial Law stipulates that "When the law requires that the information must be in writing, the data message complies with that requirement if the data contained therein can be accessed in such a way that it can be used for subsequent reference".

This is to say, the electronic check as an electronically authenticated data message is reliable and legally recognized under the UNCITRAL Act.

Article (7) paragraph (1) of the same law stipulates that: "When the law requires a person signature, that requirement shall be met in respect of the data message if:

(A) A method was used to identify that person and demonstrate that person's consent to the information contained in the data message.

(B) That method was to be relied upon to the extent appropriate for the purpose for which it was created or for which the data message was notified in the light of all circumstances, including any related agreement".

Therefore, the electronic signature included in the electronic check, which would prove the identity of the signatory and its commitment to what was signed, gives the check the force of its legal evidence. Article 10 of the same law states that "Information in the form of a data message shall be given the necessary argument of the evidence. In assessing the authentic data message in the evidence, the reliability of the method used to produce, store or communicate the data message is taken into account, to the extent that it has been used to maintain the integrity of the information, the manner in which the identity of its origin has been determined and for any relevant factor.

Since the electronic check is a data message authenticated by an electronic signature, the text of this article applies thereto (Hanan Malika, p. 91).

As for the Jordanian legislator, Article (15) of the Electronic Transactions Law (Jordan Electronic Transactions Law No. (15) of 2015) stipulates that "an electronic signature is protected if the following conditions are met:

(A) If the signatory is personalized by that signature.

(B) If it identifies the signatory.

(C) The private key is under the control of the signatory at the time of signing.

(D) If it is linked to the electronic record in a manner that does not permit an amendment to that electronic record after signing it without altering that signature".

Since the electronic check is an electronic document that contains a certified electronic signature and is used in the scope of commercial transactions, therefore it has full authoritative evidence, as the written evidence that the Jordanian legislator recognized the validity of electronic check.

The Jordanian legislator also mentioned in the Electronic Transactions Law in Article (17) paragraph (a) (Jordan Electronic Transactions Law No. (15) of 2015), that "the electronic record associated with the protected electronic signature shall have the same authenticity as the ordinary document and may be invoked by the parties to the electronic transaction".

It is noted from the previous article that the legislator granted the electronic check the power of the ordinary check in the use and evidence, provided that it is possible to save and transfer the data message (check) and it is possible to identify the parties.

The second requirement: proving the payment of the electronic check

Usually the banks when paying the check, it is received from its holder, ask him to write a term indicating the payment and he signs it. The mere receipt is a presumption of payment to prove the contrary (Dr. Mohamed Ismail, 2005, p. 322). For an electronic check, when the beneficiary receives the check, he signs it with his electronic signature as a beneficiary and sends it to the drawee bank or the bank that he deals with if it is not the drawee bank.

After confirming the identity of the beneficiary, the drawee bank shall pay through the registration in the account and send the beneficiary a file as an evidence of payment. Therefore, the drawee can refer to the electronic 
records of his accounts and the statements periodically sent to the customer as the evidence. Such records shall be considered as commercial books and shall serve as a complete evidence of proof if they are organized and the adversary is a trader (Raed Youssef, 2011, p. 130).

The Jordanian legislator considered that correspondence made during the process of payment of the check serves as a judicial presumption or as a principle of confirmation in writing.

In reference to the general rules of evidence, we note that the principle of confirmation in writing is the existence of a written paper that does not reach the level of the written evidence issued by the opponent makes the event to be proven close to the possibility. That is to say that the opponent when the completion of the principle of confirmation in writing with witnesses testimony or evidences has become fully authoritative in proving the payment by electronic check. Accordingly, the payment by electronic check can be proved by multiple evidencing methods.

\section{Conclusion}

The electronic check is the equivalent of the traditional check to include the same data as the traditional check, but it has a special particularity, since it is electronically made and mainly depends on the electronic means that have given it a special nature in trading and evidencing.

\section{Results}

1. Electronic payment methods in general and electronic check in particular is the result of the pairing between technological development and commercial life.

2. The beneficiary's name in the electronic check is one of the mandatory data contrary to the traditional check.

3. Electronic check is one of the most important results of the emergence of so-called electronic cash.

4. Nominal electronic check is the only one that accepts endorsement.

5. The electronic check is a data message authenticated by an electronic signature. Therefore, its authenticity in evidencing is subject to the electronic signature.

\section{Recommendations}

1. Providing the technological infrastructure necessary to work with electronic check as a means of electronic payment.

2. Enhancing the confidence in this tool through clear legislative texts showing the full details of the electronic check, starting with editing, through trading and ending with evidencing.

3. Enhancing the confidence of traders in this tool by providing strong guarantees in case of dealing with this check.

\section{References}

1. Jacques Hakim, Commercial Rights, Fourth Edition, Damascus University Press, 1996.

2. Essam Matar, Electronic Commerce in Arab and Foreign Legislation, New University House, Alexandria, 2009.

3. Aziz al-Ukaili, Al-Wasseet in Explaining the Commercial Law, Part II, Commercial Papers and Banking Operations, Dar Al-Thaqafa, 2010.

4. Nadia Fadil, Commercial Papers in Algerian Law, Dar Houpha Publishing, Algiers, 2006.

5. Elias Haddad, Commercial Law, Commercial Bonds, Damascus University Press, 2009.

6. Hanan Malika, Electronic Commerce, Virtual University Publications, Damascus.

7. Raed Youssef, the Legal System of Electronic Payment, Master Thesis, Algeria.

8. Mohamed Ismail, Legal Protection Methods for E-Commerce Transactions, Comparative Study, PhD Thesis, Ain Shams University, Cairo, Egypt, 2005.

9. Samer Sulaiman, the Legal System of Electronic Check, Master Thesis, Damascus University Press, 2013.

10. Musa Al Ameri, Smart Check, research presented to the Banking and Electronic Business Conference between the Sharia and Law, Volume I, UAE University, Dubai, 2003.

11. Nabil Salah Al-Arabi, Electronic Check and Digital Money, Comparative Study, study presented to the Banking and Electronic Business Conference between the Sharia and Law, United Arab Emirates University, Dubai.

12. Model Law on Electronic Signatures of the United Nations Commission on International Trade Law UNCITRAL 2001.

13. Jordan Electronic Transactions Law No. (15) of 2015.

14. Dave chaffy, E- Business and E- commerce Management, strategy, implementation and practice, Third Edition, New jersey, US, 2006

15. William s Davis and john benamati, e- commerce basics, technology- foundation and e-business application, Addison-wesley, Cornell University, newyork, 2003. 